\title{
Modified Berlekamp-Massey algorithm and modelling of discrete-time signals
}

\author{
S K MULLICK, ARUN KUMAR and PARAG JAIN* \\ Department of Electrical Engineering, Indian Institute of Technology, \\ Kanpur 208016, India \\ * P S I Data Systems Ltd. 38/A 2nd Phase, Peenya Industrial Estate, \\ Bangalore 560058, India
}

\begin{abstract}
The objective of the present paper is the study of models which view the given 1-D data as the impulse response of a minimal order discrete linear filter with data and coefficients confined to a finite field. This representation is known as "minimal partial realization" problem in linear system theory and has been tackled successfully by Ho and Kalman. Interestingly, the same problem is addressed and solved in a coding theory context by the well-known Berlekamp-Massey (BM) algorithm. We present a modified recursive version of the BM algorithm specially suited to our task of signal modelling. By introducing an absolute error bound, one can further solve the approximation problem computationally. The paper includes a number of examples and a mention of the software for this purpose.
\end{abstract}

Keywords. Discrete time signals; minimal partial realization; coding theory; signal modelling.

\section{Introduction}

The increasing predominance of digital modes of processing signals has led to an intensive study of signals and systems using discrete mathematical structures of groups, fields and vector spaces. For many signal processing tasks or even for archival storage, the raw digital representation obtained by the process of sampling and quantization is either wasteful or not ideally suited to the processing objective at hand and therefore alternate representations are often sought. When the representation is obtained via reversible transformation of the given data, this is referred to as error-free or information-preserving coding. Examples are transform domain methods, differential and run length coding. In many applications, some errors in a reconstructed signal can be tolerated and the coding schemes therefore are subject to a fidelity criterion. The most popular of these are linear prediction techniques which minimize the mean square error of representation in terms of a linear combination of a finite number of past samples. The transform domain methods have two major limitations. First, as the size of data increases so do the dimensions of the transform matrix. Second, most methods require that all data be first received before the transformation. The first 
constraint restricts us to only those transformations which can be generated recursively and the second constraint does not allow us to do real-time processing for large amount of data. The prediction schemes on the other hand avoid these constraints at the expense of a tolerable error.

The objective of the present paper is the study of models which view the given 1-D data as the impulse response of a minimal order discrete linear filter with data and coefficients confined to a finite field. The minimum partial realization problem is data-specific and provides a transfer function $B(z) / A(z)$ of a linear discrete filter with deg $B(z)<\operatorname{deg} A(z)$, and $A(z)$ having a degree which is minimal over the family of all such realizations (Kalman et al 1969). The problem may not admit of a unique solution and many such realizations may be possible. Since with each rational function $B(z) / A(z)$ we may associate a formal power series by means of long division e.g.

$$
B(z) / A(z)=s_{1} \cdot z^{-1}+s_{2} \cdot z^{-2}+\ldots,
$$

$\left(s_{1}, s_{2}, \ldots\right.$ represents the impulse response sequence), this implies that for a given finite sequence $s_{1}, \ldots, s_{n}, B(z) / A(z)$ is a realization if and only if the first $n$ coefficients of the formal power series of $B(z) / A(z)$ are exactly the numbers $s_{1} \ldots s_{n}$. In this sense, $s_{1}, \ldots, s_{n}$ may be viewed as a 'partial sequence' or a sub-sequence of a certain fixed infinite sequence and $B(z) / A(z)$ a 'partial realization'. In this sense, the problem of partial realization is the same as the Pade representation problem. To obtain minimal partial realization, we must somehow find the 'right' infinite extension sequence among the many that are possible. And in doing so we must not make any extrinsic assumptions. The problem of minimal partial realization has been tackled by a number of alternative methods. For a definitive treatment from the viewpoint of abstract algebraic system theory which is based on an algorithm due to Ho, the reader is referred to Kalman (1971). Of great relevance, however, to the present discussion is Kalman (1979) which attempts to provide an algebraic structure to all formal power series, by describing them in terms of jump points which constitute its invariants. Kalman shows, (i) that every formal power series has a representation as an infinite continued fraction, (ii) that a formal power series is rational if and only if its continued fraction representation is a terminating fraction, and (iii) that all the invariants of the power series can be recovered by expanding the rational fraction according to the Euclidean algorithm.

Interestingly enough, the same problem has been tackled in coding theory literature by the Berlekamp-Massey algorithm (Berlekamp 1968; Massey 1969; Welch \& Sholtz 1979). In fact, in a derivation of the Berlekamp-Massey algorithm based on a recursive design procedure for the shortest linear feedback shift register (LFSR) that generates a given sequence (Imamura \& Yoshide 1987), there is a complete parallel of notation of jump and jump points in terms of the behaviour of $L(n)$ vs. $n$ where $L(n)$ is the length of the shortest LFSR that generates the sequence $s_{1}, s_{2}, \ldots, s_{n} ; n<N$.

It is worth pointing out that none of the authors of references on the BM algorithm and its coding theory implications (Massey 1979; Welch \& Sholtz 1979; Imamura \& Yoshida 1987) refer to Kalman's earlier cited work (Kalman 1971, 1979) and Kalman too, fails to mention the Berlekamp-Massey algorithm connection for the scalar partial realization theory, published as late as 1979. These parallels were first mentioned in Mullick et al (1988): The application of the BM algorithm to model signals defined over finite fields was first explored by Arcese \& Reed (1984).

Our objective in this paper is to present a modified recursive BM algorithm and its software implementation for generating the minimal partial realization. A number 
of examples illustrate the use of the interactive software. It is found that, in general, the representation is as complex as the original signal, for error-free modelling, particularly for increasingly longer data sequences. This is in consonance with the answer to the questions: what is the number of minimal state deterministic linear automatons that can simulate the observed time series and how does the average number of states in the optimal model increase with number of observations (Gaines 1976; Nicolis 1986, pp. 179-181).

The significant conclusion is that deterministic finite-state automaton models provide an overcomplex view when used for simulating stochastic behaviour. However it is possible to reduce this complexity and achieve data compression using the following strategies:

1. An error bound may be incorporated and thus turn the error free realization problem to that of approximate representation.

2. A finite field autoregressive moving average (ARMA) model may be stipulated which assumes a deterministic finite state automaton excited by a suitably defined 'white noise' sequence.

\section{Modified recursive form of the Berlekamp-Massey algorithm}

We are here interested in modelling data as the impulse response of a discrete linear filter, the objective being to obtain a minimal representation by such a system. The sample domain description of a discrete linear filter is given by

$$
y_{n}=\sum_{k=0}^{M} b_{k} \cdot x_{n-k}-\sum_{k=1}^{N} a_{k} \cdot y_{n-k}
$$

The impulse response of such a filter would be

Since,

$$
y_{n}=b_{n}-\sum_{k=1}^{K} a_{k} \cdot y_{n-k}
$$

$$
\begin{aligned}
x_{n} & =1, \quad n=0 \\
& =0, \quad \text { otherwise, }
\end{aligned}
$$

therefore, (2) may be written as,

and,

$$
\sum_{k=0}^{N} a_{k} \cdot y_{n-k}=0, \quad n \geqslant N
$$

$$
\sum_{k=0}^{n} a_{k} \cdot y_{n-k}=b_{n}, \quad n=0,1, \ldots, M .
$$

The solution of the Toeplitz system of equations (3a) can be obtained by using the usual Berlekamp-Massey (BM) algorithm in any suitable field. Once the denominator coefficients, $a_{k}$ 's, are obtained, the numerator coefficients, $b_{k}$ 's, can be obtained from (3b).

A distinct disadvantage of the above procedure would be that one has to wait for half the data values to arrive before actual computation can begin. Moreover, the computation of the numerator coefficients can begin only after the denominator coefficients have been found. 
However, a recursive form of the BM algorithm (Blahut 1985) can be used to overcome the first disadvantage. Thus, one can begin computation of the denominator coefficients immediately as the data starts arriving. But one still has to wait till the denominator coefficients are calculated for these to be used along with the initial $M$ data points to obtain the numerator coefficients.

We have attempted to modify the recursive BM algorithm so that simultaneous calculations of numerator and denominator coefficients can begin once the data values start arriving, thereby eliminating both the above drawbacks.

The BM algorithm to calculate denominator coefficients is stated below:

Theorem (the BM algorithm)

In any field, let $s_{1}, s_{2}, \ldots s_{n}$ be given. Under the initial conditions $A^{0}(z)=1, L_{0}=0$, let the following set of recursive equations be used to compute $A^{n}(z)$ :

$$
\begin{aligned}
& \Delta_{r}=\sum_{j=0}^{n-1} A_{j}^{r-1} \cdot s_{r-j}, \\
& L_{r}=\delta_{r} \cdot\left(r-L_{r-1}\right)+\left(1-\delta_{r}\right) \cdot L_{r-1}, \\
& {\left[\begin{array}{l}
A^{r}(z) \\
A_{t}^{r}(z)
\end{array}\right]=\left[\begin{array}{cc}
1 & -\Delta_{r} z \\
\Delta_{r}^{-1} \delta_{r}\left(1-\delta_{r}\right) z
\end{array}\right]\left[\begin{array}{l}
A^{r-1}(z) \\
A_{t}^{r-1}(z)
\end{array}\right],}
\end{aligned}
$$

$r=1, \ldots n$, where $\delta_{r}=1$ if both $\Delta_{r}=0$ and $2 L_{r-1}<r-1$, otherwise $\delta_{r}=0$. Then, $A^{2 t}(z)$ is the smallest degree polynomial with properties that $A^{21}(z)=1$; and

$$
s_{r}+\sum_{j=1}^{n-1} A_{j}^{2 t} \cdot s_{r-j}=0, \quad j=L_{2 t+1}, \ldots 2 t
$$

To develop the recursive modified BM algorithm for simultaneous numerator and denominator updating as the data values keep arriving, we proceed as follows:

Let the transform domain representation of the filter at the $n$th stage of calculation be

$$
H^{r}(z)=B^{r}(z) / A^{r}(z)
$$

We replace the polynomials $A^{r}(z)$ and $A_{t}^{r}(z)$ in $(4 c)$ by a $2 \times 2$ matrix of polynomials:

$$
A_{M}^{r}(z)=\left[\begin{array}{ll}
A_{11}(z) & A_{12}(z) \\
A_{21}(z) & A_{22}(z)
\end{array}\right]
$$

The element $A_{i j}^{r}(z)$ is a polynomial with coefficients denoted by $A_{i j, k}^{r}$. The matrix $A_{M}^{r}(z)$ is defined such that $A^{r}(z)$ and $A_{t}^{r}(z)$ can be computed from

$$
\left[\begin{array}{l}
A^{r}(z) \\
A_{i}^{r}(z)
\end{array}\right]=\left[\begin{array}{l}
A_{11}^{r}(z)+A_{12}^{r}(z) \\
A_{21}^{r}(z)+A_{22}^{r}(z)
\end{array}\right]=A_{M}^{r}(z)\left[\begin{array}{l}
1 \\
1
\end{array}\right] .
$$

Similarly, for the numerator polynomial, create a matrix $B_{M}^{r}(z)$ as in (6) and obtain $B^{r}(z)$ and $B_{t}(z)$ from

where

$$
\left[\begin{array}{l}
B^{r}(z) \\
B_{t}^{r}(z)
\end{array}\right]\left[\begin{array}{ll}
B_{11}(z) & B_{12}(z) \\
B_{21}(z) & B_{22}(z)
\end{array}\right]=B_{M}^{r}(z)\left[\begin{array}{l}
1 \\
1
\end{array}\right],
$$

$$
B_{M}^{r}(z)=\left[\begin{array}{ll}
B_{11}^{r}(z) & B_{12}^{r}(z) \\
B_{21}^{r}(z) & B_{22}^{r}(z)
\end{array}\right] \text {. }
$$


The recursive form of the modified BM algorithm is then built from the following equations:

and

$$
\begin{aligned}
& \Delta_{r}=\sum_{j=0}^{n-1} A_{11, j}^{r-1} s_{r-j}+\sum_{j=0}^{n-1} A_{12, j}^{r-1} s_{r-j}-\left(B_{11, r}+B_{12, r}\right), \\
& A_{M}^{r}(z)=\left[\begin{array}{cc}
1 & -\Delta_{r} z \\
\Delta_{r}^{-1} \delta_{r} & \left(1-\delta_{r}\right) z
\end{array}\right] A_{M}^{r-1}(z),
\end{aligned}
$$

$$
B_{M}^{r}(z)=\left[\begin{array}{cc}
1 & -\Delta_{r} z \\
\Delta_{r}^{-1} \delta_{r} & \left(1-\delta_{r}\right) z
\end{array}\right] B_{M}^{r-1}(z)
$$

To split the algorithm into halves, suppose that $n$ is even. Let

where

$$
A_{M}^{n}(z)=A_{M}^{\prime r}(z) \cdot A_{M}^{\prime \prime n / 2}(z),
$$

$$
A_{M}^{\prime n}(z)=\prod_{r=n}^{n / 2+1}\left[\begin{array}{cc}
1 & -\Delta_{r} z \\
\Delta_{r}^{-1} \delta_{r} & \left(1-\delta_{r}\right) z
\end{array}\right]
$$

and

$$
A_{M}^{\prime \prime n / 2}(z)=\prod_{r=n / 2}^{1}\left[\begin{array}{lc}
1 & -\Delta_{r} z \\
\Delta_{r}^{-1} \delta_{r} & \left(1-\delta_{r}\right) z
\end{array}\right]
$$

Similarly

$$
B_{M}^{n}(z)=B_{M}^{\prime n}(z) \cdot B_{M}^{\prime n / 2}(z)
$$

where

$$
B_{M}^{\prime n}(z)=\prod_{r=n}^{n / 2+1}\left[\begin{array}{cc}
1 & -\Delta_{r} z \\
-\Delta_{r}^{-1} \delta_{r} & \left(1-\delta_{r}\right) z
\end{array}\right]
$$

and,

$$
B_{M}^{\prime n / 2}(z)=\prod_{r=n / 2}^{1}\left[\begin{array}{cc}
1 & -\Delta_{r} z \\
-\Delta_{r}^{-1} \delta_{r} & \left(1-\delta_{r}\right) z
\end{array}\right]
$$

The two halves are computed separately and multiplied together. Now, $\Delta_{r}$ can be thought of as the nth coefficient of the first component of the two vectors of polynomials:

$$
\left[\begin{array}{c}
\Delta(z) \\
\Delta^{\prime}(z)
\end{array}\right]=\left[\begin{array}{ll}
A_{11}^{r-1}(z) & A_{12}^{r-1}(z) \\
A_{21}^{r-1}(z) & A_{22}^{r-1}(z)
\end{array}\right]\left[\begin{array}{c}
s(z) \\
s(z)
\end{array}\right]-\left[\begin{array}{l}
B_{11}(z)+B_{12}(z) \\
B_{21}(z)+B_{22}(z)
\end{array}\right] .
$$

Hence, for $r>n / 2$, we have

where

$$
\begin{aligned}
{\left[\begin{array}{c}
\Delta(z) \\
\Delta^{\prime}(z)
\end{array}\right] } & =A_{M}^{r-1}(z) \cdot A_{M}^{\prime r-1}(z)\left[\begin{array}{l}
s(z) \\
s(z)
\end{array}\right]-\left[\begin{array}{l}
B_{11}^{r-1}(z)+B_{12}^{r-1}(z) \\
B_{21}^{r-1}(z)+B_{22}^{r-1}(z)
\end{array}\right] \\
& =A_{M}^{r-1}(z) \cdot S_{M}^{n / 2}(z)-\left[\begin{array}{l}
B_{11}^{r-1}(z)+B_{12}^{r-1}(z) \\
B_{21}^{r-1}(z)+B_{22}^{r-1}(z)
\end{array}\right]
\end{aligned}
$$

$$
S_{M}^{n / 2}(z)=A_{M}^{\prime \prime n / 2}(z)\left[\begin{array}{l}
s(z) \\
s(z)
\end{array}\right]
$$

Based on the above, the recursive form of the modified BM algorithm can be stated in terms of $(10 a),(10 b)$ and $(10 c)$ as follows:

$$
\Delta_{r}=\sum_{j=0}^{n-1} A_{11, j}^{r-1} \cdot s_{1, r-j}+\sum_{j=0}^{n-1} A_{12, j}^{r-1} \cdot s_{2, r-j}-\left(B_{11, r}+B_{12, r}\right),
$$




$$
A^{r}(z)=\left[\begin{array}{cc}
1 & -\Delta_{r} z \\
\Delta_{r}^{-1} \delta_{r} & \left(1-\delta_{r}\right) z
\end{array}\right] A^{r-2}(z)
$$

and,

$$
B^{r}(z)=\left[\begin{array}{cc}
1 & -\Delta_{r} z \\
\Delta_{r}^{-1} & \delta_{r} \\
\left(1-\delta_{r}\right) z
\end{array}\right] B^{r-1}(z),
$$

where $A^{r}(z)$ may represent either $A^{\prime r}(z)$ or $A^{\prime \prime r}(z), B^{r}(z)$ may represent either $B^{\prime \prime}(z)$ or $B^{\prime \prime r}(z)$, and $\left(S_{1}(z), S_{2}(z)\right)$ represents $(S(z), S(z))$ in the first half of the computation and is updated to $S_{M}^{n / 2}(z)$ in the second half. After the calculation of both the halves are complete, $A^{n}(z)$ and $B^{n}(z)$ are obtained by multiplying the two halves.

After the modified BM algorithm is split into two halves, each half itself is a BM

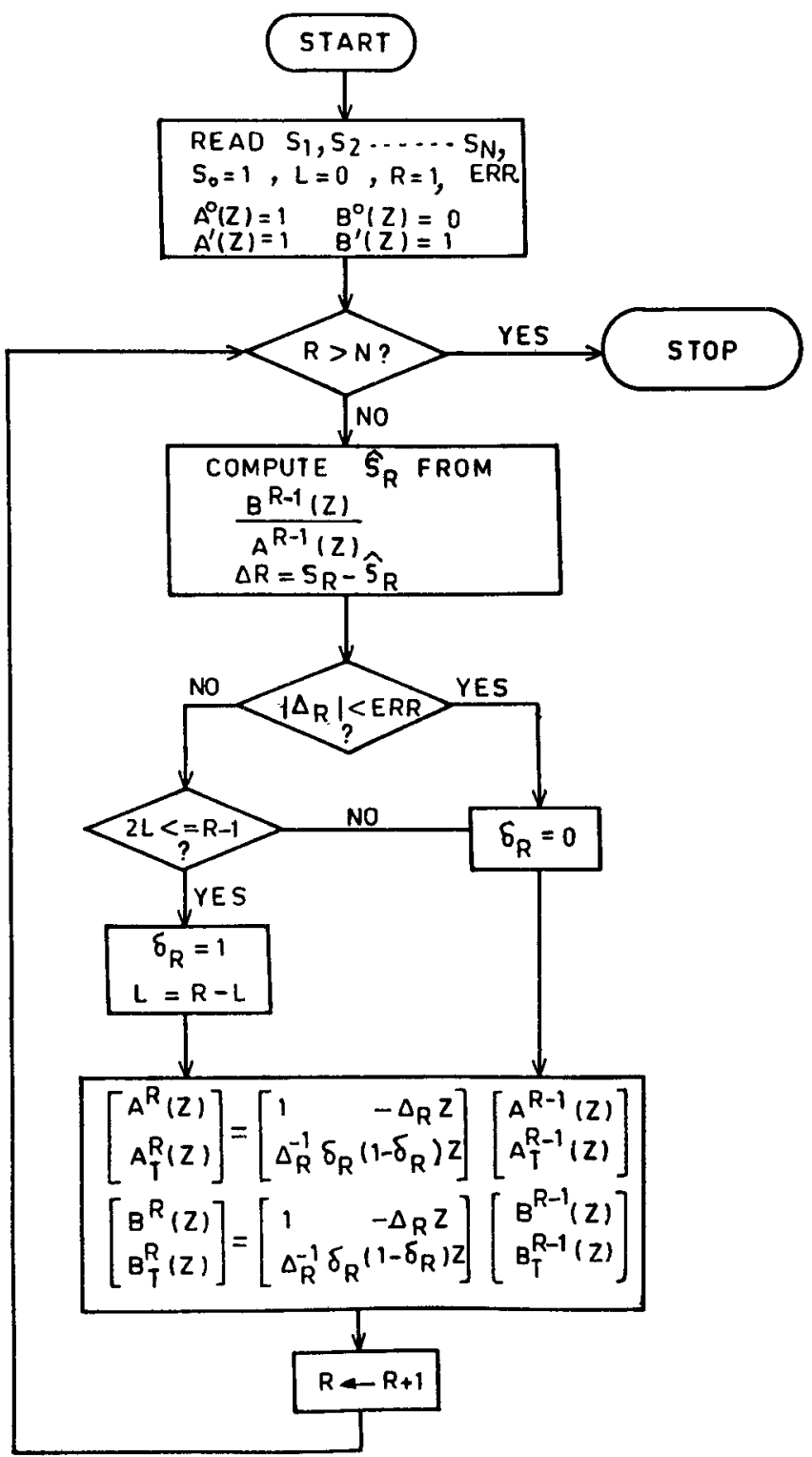

Figure 1. Modified recursive BM algorithm with error bound. 
algorithm. Hence, if $n / 2$ is even, the two halves can in turn be split. If $n$ is a power of two, then the splitting can be continued until we reach a stage of one-iteration long computations. This introduces the maximum computational efficiency.

Initialization of the matrices is done as follows: Let $\Delta_{1}$ be the error between $S_{1}$ and $S_{2}$. Then

$$
A^{1}(z)=\left[\begin{array}{cc}
1 & -\Delta_{1} z \\
\Delta_{1}^{-1} \delta_{1} & \left(1-\delta_{1}\right) z
\end{array}\right], \quad \text { and } \quad B^{1}(z)=\left[\begin{array}{cc}
1 & -\Delta_{1} z \\
\Delta_{1}^{-1} \delta_{1} & \left(1-\delta_{1}\right) z
\end{array}\right] \text {. }
$$

The algorithm is depicted in figures 1 and 2. Figure 1 shows recursive procedure of the modified BM algorithm, while figure 2 shows the speeded-up version. The latter

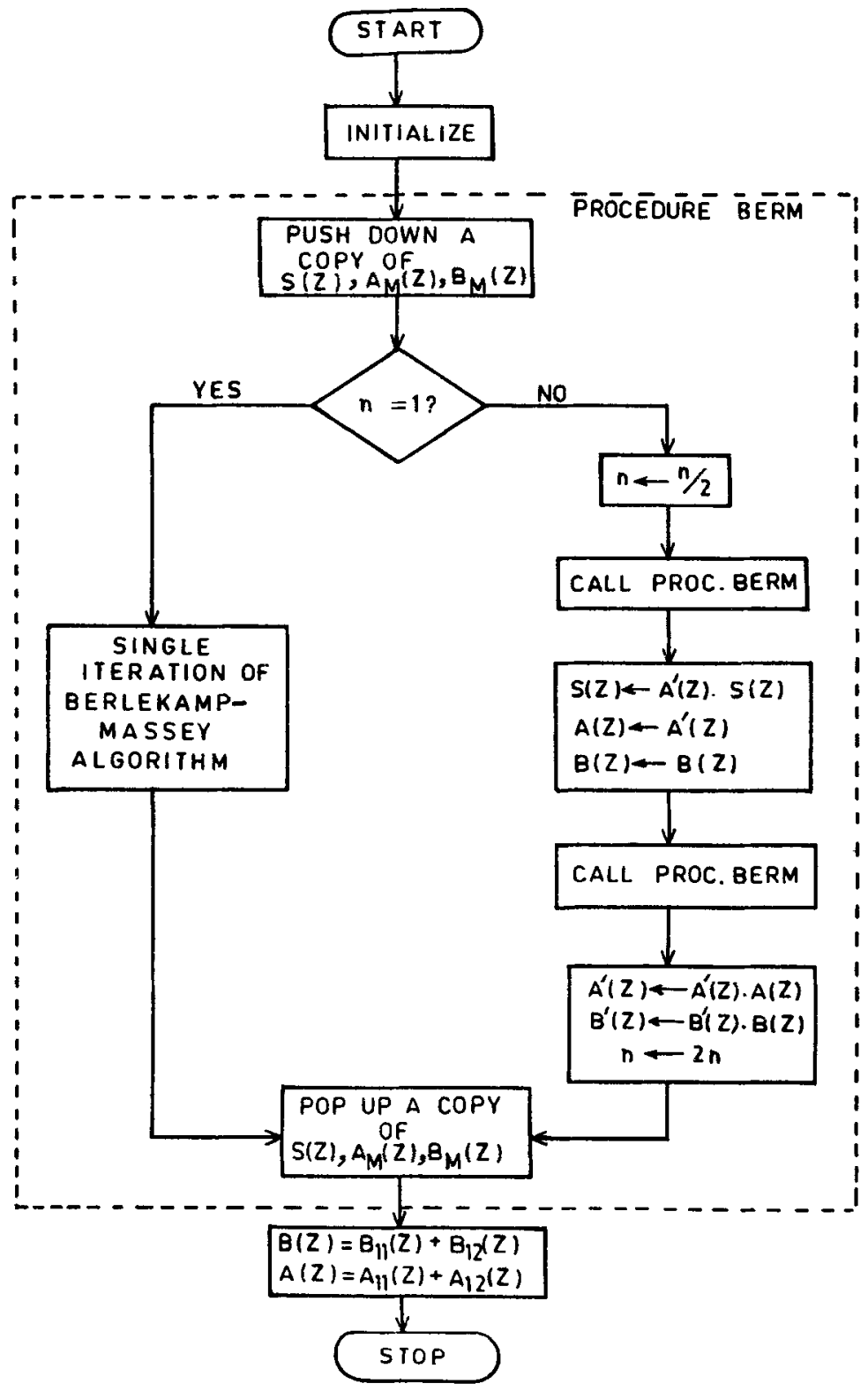

Figure 2. Faster version of modified BM algorithm. 
is advantageous in the sense that it takes advantage of the simplicity of early iterations by doing two iterations at a time.

\section{Introduction of error bound}

The above algorithm gives a transfer function realization of a filter which when excited by an impulse, regenerates the data exactly. It must be noted that at the rth stage, if we can use the transfer function,

$$
H^{r}(z)=B^{r}(z) / A^{r}(z)
$$

to calculate $s_{r+1}$, the predicted $(r+1)$ th value of the data, and it turns out to be equal to $s_{r+1}$, i.e., if the error $\Delta_{r+1}=0$, then

$$
H^{r+1}(z)=H^{r}(z)
$$

and we forego the usual multiplication process for the $(r+1)$ th stage. This rarely occurs in a practical situation. If we, however, allow for an error in the predicted values, such that if

$$
\Delta_{r+1}=\left|s_{r+1}-\hat{s}_{r+1}\right|<\text { maximum allowable error, }
$$

then we substitute $\Delta_{r+1}=0$ and do not modify $H^{r}(z)$. The maximum error must, however, be less than one quarter the modulus field of operation (Arcese \& Reed 1984). In such a case, the calculation can be done faster provided the error found on the reconstructed data points is acceptable.

\section{Software implementation}

We have implemented the speeded-up version of the modified BM algorithm in Turbo Pascal. It is a user-interactive program. The program INVERSE creates a file TABLE containing the inverse values of all the elements in the chosen field of operation. This program should be executed before the main program BM is executed.

The program BM asks for the following information from the user in the given format:

Give the size of the modulo field-

Give the number of input values-

TABLE contains the inverse values of the modulo field.

Does TABLE exist in the directory? Type $\mathrm{Y}$ or $\mathrm{N}$ -

(If the user types $\mathrm{N}$ then the program is terminated and the user should create TABLE by first running INVERSE. If $\mathrm{Y}$ is typed then the program proceeds.)

Give the tolerable error bound-

The input values should be entered in the file INPUT. Correspondingly, the output is stored in the OUTPUT file. The format of the output file is as follows:

Error bound

Numerator coefficients

Denominator coefficients

Predicted sequence. 


\section{Examples and results}

(i) Error bound =0,

Modulo field $=97$,

Desired sequence: $16,10,37,52,55, \ldots$,

Transfer function obtained using the algorithm

$$
H(z)=\left(1+84 z+9 z^{2}\right) /\left(1+68 z+75 z^{2}+23 z^{3}\right) .
$$

(ii) Error bound $=15$,

Modulo field $=97$,

Desired sequence: Same as above.

Transfer function obtained using BM algorithm with error bound

$$
H(z)=1 /(1+77 z)
$$

Generated sequence: $20,12,46,47,67, \ldots$,

Error sequence: $4,2,9,5,12, \ldots$.

(iii) Example (Kalman 1969, 1971)

Desired sequence $1,1,1,2,1,3, \ldots$,

Error $=0$,

Unique minimal transfer function obtained in $[1,2]$ is

$$
H(z)=\left(z^{2}+2 z+1\right) /\left(z^{3}+z^{2}-z-2\right) .
$$

The same sequence realized by the BM algorithm using a modulo field 5 is

$$
H(z)=\left(1+2 z+z^{2}\right) /\left(1+z+4 z^{2}+3 z^{3}\right) .
$$

The two results, though they seem different, are actually identical. The different appearance is due to the fact that in Kalman, the formal power series associated with $H(z)$ is in variable $z^{-1}$ instead of $z$, and that he uses the field of integers.

\section{Conclusion}

We have explored the possibility of using the BM algorithm for representing discrete signals defined over finite fields. For this purpose a modified recursive BM algorithm has been derived for simultaneous updating of numerator and denominator coefficients of the discrete transfer function, as data values are received. An interactive software in Turbo Pascal for implementing this algorithm with an incorporated error constraint has been written. Experimentation has shown that for lossless coding, the representation is almost as complex as the original data. This is in conformity with the results from complexity theory of deterministic optimal linear finite state machines models for simulating a time series. For data compression using this approach, one will have to either incorporate an error bound or devise a finite field ARMA model which assumes an excitation by a suitably defined 'white noise' sequence. This needs further exploration. Moreover these ideas need extension to 2-D signals for image models. It has come to the author's knowledge that this has recently been achieved by Sakata (1990) by deriving a 2-D version of the BM algorithm. 


\section{References}

Arcese A, Reed I S 1984 Linear prediction on a Galois field, digital signal processing-84 (eds) V Cappellini, A G Constantinides (Amsterdam: North Holland/Elsevier Science Publishers.)

Berlekamp E R 1968 Algebraic coding theory (New York: McGraw Hill)

Blahut R E 1985 Fast algorithms for digital signal processing (Reading, Mass: Addison Wesley) chap. 11

Gaines BR 1976 On the complexity of causal models. IEEE Trans. Syst., Man Cybern. 6: 56-59

Imamura K, Yoshide W 1987 A simple derivation of the Berlekamp-algorithm and some applications. IEEE Trans. Inf. Theory IT-33: 146-150

Kalman R E 1971 On minimal partial realization of a linear input/output map. Aspects of network and system theory (eds) R E Kalman, N DeClaris (New York: Holt, Rinehart and Winston)

Kalman R E 1979 On partial realizations, transfer functions and canonical forms. Acta Polytech. Scand. 31: $9-32$

Kalman R E, Falb P E, Arbi M A 1969 Topics in mathematical system theory (New York: McGraw Hill) chap. 10

Massey J L 1969 Shift register synthesis and BCH decoding. IEEE Trans. Inf. Theory IT-15: 122-127

Mullick S K, Jain P, Kumar A 1988a Partial realization, Berlekamp-Massey algorithm and modelling of discrete time signals. IEEE Digital Signal Processing Workshop, USA, 4.7.1-4.7.2

Mullick S K, Rastogi R, Mullick R 1988b Partial realization, Berlekamp-Massey algorithm and image coding. Indo-US Workshop on Systems and Signal Processing, Bangalore.

Nicolis J S 1986 Dynamics of hierarchical systems (Berlin: Springer Verlag) chap. 4, \$4.6

Sakata S 1990a Partial realization of 2-D discrete linear system and 2-D Pade' approximation and reduction of 2-D rational transfer function. Proc. IEEE 78: 604-613

Sakata S 1990b Extension of the Berlekamp-Massey algorithm to $N$ dimensions. Inf. Comput. 84: 207-239

Welch L R, Sholtz R A 1979 Continued fraction and Berlekamp's algorithm. IEEE Trans. Inf. Theory IT-25: 19-27 